\title{
BMJ Open UK Chiari 1 Study: protocol for a prospective, observational, multicentre study
}

\begin{abstract}
Rory J Piper (D , ${ }^{1}$ Fardad T Afshari, ${ }^{2}$ Wai Cheong Soon, ${ }^{2}$ Angelos G Kolias, ${ }^{3,4}$ Edward W Dyson (D) , ${ }^{5}$ Laurence Watkins (D) , ${ }^{5}$ Rodney Laing, ${ }^{3,4}$ William B Lo, ${ }^{6}$ Jayaratnam Jayamohan, ${ }^{1}$ the British Neurosurgical Trainee Research Collaborative (BNTRC)
\end{abstract}

To cite: Piper RJ, Afshari FT, Soon WC, et al. UK Chiari 1 Study: protocol for a prospective, observational, multicentre study. BMJ Open 2021;11:e043712. doi:10.1136/ bmjopen-2020-043712

- Prepublication history and additional materials for this paper is available online. To view these files, please visit the journal online (http://dx.doi org/10.1136/bmjopen-2020043712).

Received 12 August 2020 Revised 30 November 2020 Accepted 10 March 2021
Check for updates

(C) Author(s) (or their employer(s)) 2021. Re-use permitted under CC BY-NC. No commercial re-use. See rights and permissions. Published by BMJ.

For numbered affiliations see end of article.

Correspondence to

Rory J Piper;

rorypiper@doctors.org.uk

\section{ABSTRACT}

Introduction Chiari 1 malformation (CM1) is a structural abnormality of the hindbrain characterised by the descent of the cerebellar tonsils through the foramen magnum. The management of patients with $\mathrm{CM} 1$ remains contentious since there are currently no UK or international guidelines for clinicians. We therefore propose a collaborative, prospective, multicentre study on the investigation, management and outcome of CM1 in the UK: the UK Chiari 1 Study (UKC1S). Our primary objective is to determine the health-related quality of life (HRQLL) in patients with a new diagnosis of $\mathrm{CM} 1$ managed either conservatively or surgically at 12 months of follow-up. We also aim to: (A) determine HRQoL 12 months following surgery; (B) measure complications 12 months following surgery; (C) determine the natural history of patients with $\mathrm{CM} 1$ treated conservatively without surgery; (D) determine the radiological correlates of presenting symptoms, signs and outcomes; and (E) determine the scope and variation within UK practice in referral patterns, patient pathways, investigations and surgical decisions.

Methods and analysis The UKC1S will be a prospective, multicentre and observational study that will follow the British Neurosurgical Trainee Research Collaborative model of collaborative research. Patients will be recruited after attending their first neurosurgical outpatient clinic appointment. Follow-up data will be collected from all patients at 12 months from baseline regardless of whether they are treated surgically or not. A further 12-month postoperative follow-up timepoint will be added for patients treated with decompressive surgery. The study is expected to last three years.

Ethics and dissemination The UKC1S received a favourable ethical opinion from the East Midlands Leicester South Research Ethics Committee (REC reference: 20/ EM/0053; IRAS 269739) and the Health Research Authority. The results of the study will be published in peer-reviewed medical journals, presented at scientific conferences, shared with collaborating sites and shared with participant patients if they so wish.

\section{BACKGROUND}

Chiari 1 malformation (CM1) is a structural abnormality of the hindbrain characterised by the descent of the cerebellar tonsils
Strengths and limitations of this study

- The UK Chiari 1 Study (UKC1S) will be a prospective multicentre study of the management of patients with Chiari 1 malformation.

- Contrary to other studies which focus on surgical management only, the UKC1S will study patients who have been managed with and without surgery.

- The UKC1S is adopting a trainee-led study process that has been shown to be deliverable and effective.

- This study will offer an insight into the contemporary trends in the neurosurgical management of Chiari 1 malformation in the UK.

- The study uses different quality of life measures for adults and children, so no direct comparison between these groups will be possible.

through the foramen magnum. It is one of six Chiari malformations described, ${ }^{1}$ but is the most common of these and has an estimated prevalence of approximately 8 in 1000 people. ${ }^{2}$ CM1 affects patients of all ages, but more commonly presents in late childhood or early adulthood.

CM1 is a heterogeneous condition. The natural history of CM1 in some patients is relatively benign and some patients remain asymptomatic. ${ }^{3}$ Other patients with CM1 may have significant symptoms, neurological deficits and complications. ${ }^{4}$ Crowding of the neural structures in the foramen magnum can present with brainstem, cerebellar and spinal cord symptoms. There may be disruption to the normal flow of cerebrospinal fluid (CSF) through the foramen magnum and $50 \%$ of imaged patients have an associated spinal cord syrinx, and a small number of patients develop hydrocephalus. ${ }^{5}$

Despite the Chiari malformations being classified in $1891,{ }^{6}$ the aetiology, classification, diagnosis, natural history, investigation and management of CM1 remain as highly 
contentious subjects. There are currently no UK or international guidelines for the investigation or management of CM1. ${ }^{7}$

Surgery to decompress the craniocervical junction (often termed foramen magnum decompression (FMD)) remains the mainstay of surgical management. The surgical technique for decompressive surgery varies widely between centres and surgeons. ${ }^{8} 9$ FMD can comprise 'bone-only decompression', but can also include dural opening, arachnoid opening, duraplasty and tonsillar resection. Recent evidence suggests that there may be differences in efficacy and outcomes between these techniques, ${ }^{10}{ }^{11}$ but further evidence is required. The use of intraoperative technologies, such as ultrasonography, to inform surgical decisions also requires further evidence of efficacy. ${ }^{12}$ CSF diversion has also been advocated as a possible primary strategy for the management of symptomatic CM1 with evidence of abnormal intracranial pulsatility. ${ }^{13}$

Current evidence in CM1 consists mostly of relatively small, single-centre and/or retrospective studies. While evidence in CM1 is available on a national scale in other countries ${ }^{14}$ there are no UK-wide studies that have reported or compared the scope or variation in contemporary neurosurgical workload, practice and outcomes. Multicentre US databases have offered larger scale analyses of surgical outcomes, but these are retrospective and only focus on surgically managed patients. ${ }^{915}$ While there are some retrospective studies, ${ }^{3}{ }^{16}$ there is a distinct lack of prospective studies investigating outcomes in conservatively managed patients. The lack of evidence in this field makes it difficult for surgeons to decide which patients surgery should be offered and which patients can be managed without surgery. There is a clear need for largescale and non-biased data and we therefore propose a collaborative, multicentre and prospective study of the investigation and management of CM1 in the UK.

\section{METHODS \\ Objectives}

The primary objective of the study is to determine the change in health-related quality of life (HRQoL) between the first neurosurgical clinic appointment and at 12 months of follow-up of patients with CM1. Our secondary objectives are to: (A) determine HRQoL at 12 months following surgery; (B) measure complications and need for reoperation within 12 months of surgery; (C) determine the natural history of patients with CM1 treated conservatively without surgery; (D) determine the radiological correlates of presenting symptoms, signs and outcomes; and (E) determine the scope and variation within UK practice in referral patterns, patient pathways, investigations and surgical decisions.

\section{Design}

The UK Chiari 1 Study (UKC1S) will be a prospective, multicentre, observational study. The study is purely observational and will not alter patient care in any way.

The study will follow the British Neurosurgical Trainee Research Collaborative (BNTRC) model for multicentre and trainee-led collaboration. ${ }^{17}$ Each participating UK neurosurgical unit (NSU) will be required to commit both a consultant principal investigator (PI) and a trainee co-PI. As many interested consultants as possible are welcome to participate as collaborators. We are particularly interested in the collaboration of consultant neurosurgeons who have an interest in CM1 and who see substantial numbers of patients with CM1. Considering the length of the study, trainee investigators may be required to hand over the trainee co-PI role to another trainee during the study course, but this will not interfere with the study design. All UK NSUs will be invited to participate.

Each participating NSU is permitted to begin a 12-month recruitment period between 8 October 2020 and 7 April 2021. The overall study, therefore, will be completed on 6 April 2024.

\section{Inclusion criteria}

Participants will be patients with a new diagnosis of CM1 presenting to neurosurgical clinic for the first time within the one year recruitment stage of the study. We will recruit both adults and children of any age to the study. While at least $5 \mathrm{~mm}$ of herniation below the foramen magnum is often used to decide the diagnosis of CM1, such an arbitrary threshold is not specified in this study. Since this is a pragmatic study, we instead choose to include all patients who have been diagnosed with CM1 by their consulting neurosurgeon.

\section{Exclusion criteria}

Patients may not enter the study if they are considered to have an alternative Chiari malformation, history of spinal dysraphism or if they have already undergone neurosurgical intervention (excluding lumbar puncture) for CM1 or an alternate neurological diagnosis.

\section{Outcome measures}

The study will collect both patient-reported or parentreported (hereafter referred to simply as patientreported) and surgeon-reported data. The study design allows for remote, online data collection and therefore does not require patients to make further physical visits to the hospital or any other facility. Participants will be required to provide patient-reported data at baseline and 12 months following baseline (figure 1 and table 1). Additionally, if the patient undergoes decompressive surgery, then they will be asked to provide patient-reported data 12 months after their surgery. This patient-reported model of data collection has been piloted and shown to be feasible by the Understanding Cauda Equina Study. ${ }^{18}$ Surgeon-reported data will require observational data collection from patient notes, imaging and surgical logs. 


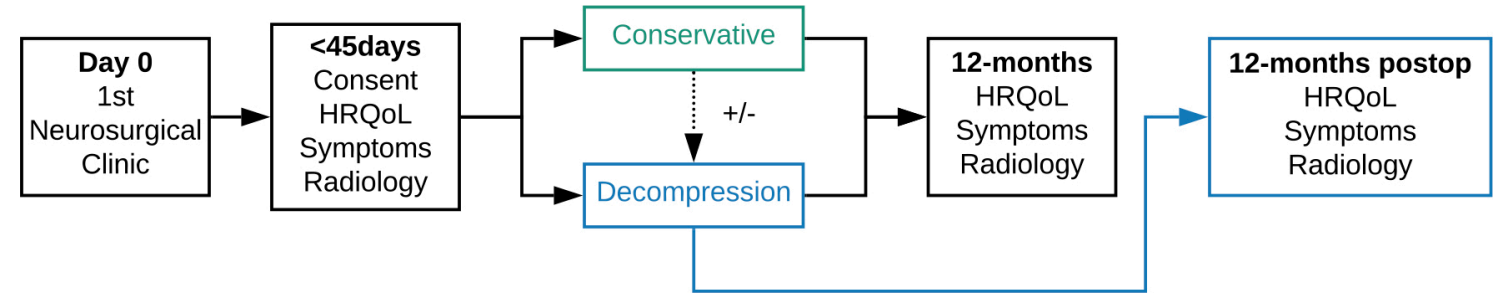

Figure 1 Study process. HRQoL, health-related quality of life.

The data fields for collection are available as online supplemental material.

Patient-reported data at baseline, 12 months and, if applicable, 12 months following surgery will consist of an online questionnaire regarding symptoms and HRQoL. HRQoL will be measured in adults (16 years and above) using the 36-Item Short Form Health Survey (UK version). HRQoL will be measured in children (2-15 years) using the age-appropriate parent-reported Pediatric Quality of Life Inventory (PedsQL; UK version). Written permission was given by the Mapi Research Trust (www.mapi-trust. org) to use the PedsQL questionnaires free of charge. Children less than twoyears of age will be recruited into the study, but not included in the HRQoL analyses.

\section{Screening and consent}

Patients who satisfy all of the inclusion and exclusion criteria will be identified by participating neurosurgical consultants and trainees. The neurosurgical team will ask permission for the patient to be contacted by the study team (ie, the researchers working on the study at that particular hospital/NSU) via telephone and this will be documented in the patient's notes.

After identification, patients will be contacted via telephone by a member of the study team who will explain the details of the study, its aims and what it involves for the participant. The person who obtained the consent must be suitably qualified and experienced and has been authorised to do so by the principal investigator. The study will be explained and if the patient agrees then the patient will be sent an email that contains a PDF version of the participant information sheet and a hyperlink to

\begin{tabular}{|c|c|c|c|}
\hline Procedures & Day 0 & $\begin{array}{l}12 \\
\text { months }\end{array}$ & $\begin{array}{l}12 \text { months } \\
\text { postoperative }\end{array}$ \\
\hline $\begin{array}{l}\text { First neurosurgical } \\
\text { clinic }\end{array}$ & $\checkmark$ & & \\
\hline Patient identified & $\checkmark$ & & \\
\hline Patient approached & $\checkmark$ & & \\
\hline Patient consented & $\checkmark$ & & \\
\hline $\begin{array}{l}\text { Patient-reported } \\
\text { data }\end{array}$ & $\checkmark$ & $\checkmark$ & $\checkmark$ \\
\hline $\begin{array}{l}\text { Surgeon-reported } \\
\text { data }\end{array}$ & $\checkmark$ & $\checkmark$ & $\checkmark$ \\
\hline
\end{tabular}

*Only for patients undergoing decompressive surgery. the digital consent form. The digital consent form will be a submission form linked to our Research Electronic Data Capture (REDCap) study database. Patients will explicitly be consented for their name, email address and phone number to be stored on the database. Patients will draw an electronic signature, type their name and type the date on the electronic consent form.

The parents or legal guardians will consent for children ( $<16$ years) approached for the study and the parents' contact details will be used. If a child turns 16 years of age during the study period, then the patient will be reconsented to remain in the study using the same procedures as described for adults. Those who are unable to consent to the study themselves may be enrolled by their next of kin.

At the time of consent, an additional option will be for patients to consent to being contacted for future studies. We acknowledge that a one-year follow-up is too short in order to determine the outcomes and reveal the management decisions in this long-term condition, but the study timeline is pragmatically built on the collaborative BNTRC model. We plan, however, for a future study to collect outcome data at five and/or 10 years, but these are considered separate and are not within the current study protocol.

During the course of the study a participant may choose to withdraw early from the study at any time.

\section{Statistical analysis plan}

The study outcomes will be measured using quantitative data. There is no qualitative component to the study.

For the primary outcome, the baseline and 12-month HRQoL assessments will be used for analysis. For all patients, comparison will be made between baseline and 12 months, but both will also be compared against the UK normative data sets. For the secondary outcome in the subgroup undergoing decompressive surgery, HRQoL data will be similarly compared with baseline and to UK normative data sets. Adult and paediatric HRQoL will be analysed separately since the measurement tools are different. For each follow-up timepoint, patient-reported data (digital questionnaires) must be completed within 45 days from the target date to be included in the analysis.

Further comparisons of outcome data will be made between specific surgical techniques used, baseline symptoms and radiological findings. Descriptive statistics will be used to report the data from the exploratory objectives, regarding referral pathways, investigations and 
management strategies for CM1 in UK neurosurgical practice.

Statistical significance will be set at $\mathrm{p}<0.05$.

A pilot retrospective data review from two NSUs (Birmingham and Oxford) showed that, in onemonth, one unit found 11 patients and the other found four patients who met the inclusion criteria aforementioned. Based on these limited data, if 18 participating units saw this average of seven patients per month, over the 12-month recruitment periods there would be around 1500 eligible patients. Considering that not all patients will agree to take part and that some patients may be missed, we realistically aim for a recruitment target of at least 500 patients.

\section{Patient and public involvement}

The study design has been presented to and has received feedback from the Ann Conroy Trust, a charity and support group for patients with Chiari malformations and syringomyelia. Patients will be given the additional option on the consent form to be contacted following the completion of the study for the synopsis of the overall study results to be emailed to the email address they provided.

\section{ETHICS AND DISSEMINATION \\ Approvals}

Oxford University Hospitals NHS Foundation Trust will act as the study sponsor. Approval has been granted by the East Midlands Leicester South Research Ethics Committee (REC reference: 20/EM/0053; IRAS 269739) and the Health Research Authority. Each NSU will require written approval from their local Research and Development department.

The UKC1S was selected for support by the BNTRC following an open national call for projects in 2018. It has been reviewed by the BNTRC Committee, the Society of British Neurological Surgeons Academic Committee and the British Syringomyelia Chiari Group.

Quality assurance procedures will be conducted by the sponsor site. The study may be monitored, or audited in accordance with the current approved protocol, Good Clinical Practice, relevant regulations and standard operating procedures.

The investigators will ensure that this study is conducted in accordance with the principles of the Declaration of Helsinki. The investigators will ensure that this study is conducted in accordance with relevant regulations and with Good Clinical Practice. While it is not an absolute requirement, we would encourage all collaborators to complete their Good Clinical Practice training prior to the start of their involvement in the study.

\section{Data management}

Data will be entered onto a secure online database platform called REDCap (https://projectredcap.org/). ${ }^{19}$ REDCap has been used extensively in clinical studies by the National Health Service (NHS) and UK universities, including recent studies by the STARSurg collaborative. ${ }^{20}$ Online data capture has been used successfully by previous BNTRC studies, such as the Understanding Cauda Equina Study. ${ }^{18}$

All information will be kept strictly confidential and the study will comply with the Data Protection Act 1998 and the European Union General Data Protection Regulation (GDPR).

Every member of the local study teams will be given personal log-in details in order to input data into the database. Local study team members will have full access to their local data, but not the data of other participating NSUs. The sponsor site will have full access to all of the data (from every NSU) to allow multicentre data analysis, ensure ongoing validity of the study or to follow-up with patients. The REDCap system also allows the sponsor site full visibility of the electronic audit trail that tracks all user activity on the database, allowing full accountability of its usage and to ensure standard operating procedures are maintained.

Once a patient electronically signs the consent form, the REDCap system will automatically assign the patient a unique UKC1S ID number. Each NSU will keep a log of the corresponding local ID number or NHS number on their local secure NHS system. With their consent, each patient's name, email address and phone number will be securely stored on the database and are hidden behind access controls so that only the local site and sponsor site can see them. Other than on the consent form, the name and any other identifying detail will not be included in any study data electronic file.

Following completion of the study, all data recorded on the database will be downloaded by the sponsor site and retained within the secure electronic NHS system for a maximum of 10 years following completion of the study. Paper documentation will be retained in appropriately secure NHS storage and electronic data will be stored on the sponsor NHS server. Data, including the separately stored consent forms, will be retained further if patients have been consented to being contacted for future studies.

\section{Data confidentiality}

The study will comply with the GDPR and Data Protection Act 2018, which require data to be deidentified as soon as it is practical to do so. The processing of the personal data of participants will be minimised by making use of a unique participant study number only on all study documents and any electronic database(s), with the exception of the consent form where the patients must type their name and sign the form. All documents will be stored securely and only accessible by study staff and authorised personnel. The study staff will safeguard the privacy of participants' personal data.

\section{Funding}

This study is kindly supported by the Ann Conroy Trust, primarily a support group for those affected byChiari 
malformations, syringomyelia and associated conditions (www.annconroytrust.org).

\section{Dissemination plan}

We intend to publish and present our study outcomes in peer-reviewed medical journals and conferences, respectively. In publications including national data, as per the BNTRC $^{17}$ and Association of Surgeons in Training $(\mathrm{ASiT})^{21}$ models, all participating researchers will be named as PubMed-citable collaborators. The REDCap database allows visibility of data input and will ensure that named collaborators have contributed towards the study. Authorship will be decided in accordance with the International Committee of Medical Journal Editors. As per the BNTRC constitution, the senior author will be the BNTRC (https://www.bntrc.org.uk/about-us). The steering committee and BNTRC will retain ownership of the entire national data set and the right to publish the data. Local data, however, will be available to the local investigators to facilitate local audit. Local outputs must acknowledge the UKC1S. This study is intended as a research study in order to inform further research and perhaps future clinical practice. Variance in practice or the outcomes of individual NSU or neurosurgeon will be available for each NSU to review, but NSU or individual neurosurgeon identifying data will not be exposed publicly or to any other NSU.

\section{Author affiliations}

${ }^{1}$ Department of Neurosurgery, John Radcliffe Hospital, Oxford, UK

${ }^{2}$ Department of Neurosurgery, Queen Elizabeth Hospital, Birmingham, UK

${ }^{3}$ Division of Neurosurgery, Department of Clinical Neurosciences, University of Cambridge, Cambridge, UK

${ }^{4}$ Division of Neurosurgery, Addenbrooke's Hospital, Cambridge, UK ${ }^{5}$ Department of Neurosurgery, National Hospital for Neurology and Neurosurgery, University College London Hospitals NHS Foundation Trust, London, UK ${ }^{6}$ Department of Neurosurgery, Birmingham Children's Hospital NHS Foundation Trust, Birmingham, UK

Acknowledgements RJP was supported by an NIHR Academic Clinical Fellowship. AGK is supported by a Clinical Lectureship, University of Cambridge and the Royal College of Surgeons of England.

Collaborators The British Neurosurgical Trainee Research Collaborative (BNTRC): Aabir Chakraborty; Adrian Casey; Adriana Baritchii; Ahmed Toma; Alexandros Vyziotis; Ali Nader-Sephai; Alistair Jenkins; Amin Andalib; Anan Shtaya; Andrew Alalade; Andrew Brodbelt; Ardalan Zolnourian; Arup Ray; Asfand Baig Mirza; Ashwin Kumaria; Aswin Chari; Babar Vaqas; Barrie White; Benedetta Pettorini; Chandrasekaran Kaliaperumal; Daniel Thompson; Danyal Khan; Dardis Ronan; David Choi; David Rowland; Dominic Thompson; Edward Jerome St George; Eleni Maratos; Georgios Tsermoulas; Grainne McKenna; Greg James; Hani Marcus; Hasan Asif; Hugo Layard Horsfall; Ian Kamaly-Asl; Ibrahim Jalloh; James Stewart; Jawad Naushahi; Joe M Das; John Duddy; Jonathan Funnell; Joseph Merola; Jothy Kandasamy; Julie Woodfield; Justyna Ekert; Kevin Tsang; Lewis Thorne; Linda D’Antona; Lizkerry Odeh; Louise Young; Makinah Haq; Mano Shanmuganathan; Mansoor Foroughi; Marianne Hare; Mark Nowell; Matthew Boissaud-Cooke; Melissa Gough; Menaka Paranathala; Micaela Uberti; Michael Cearns; Milan Makwana; Milo Hollingworth; Ming Yao Chong; Muhammad Kamal; Musa China; Nadia Salloum; Navneet Singh; Nicholas Haden; Nikolaos Tzerakis; Oscar MacCormac; Pasquale Gallo; Paul Leach; Peter McGarrity; Rhannon Lobo; Richard Edwards; Rosa Sun; Rudrajit Kanjilal; Ryan Waters; Saeed Kayhanian; Samuel Jeffery; Saurabh Sinha; Setthasorn Zhi Yang 0oi; Shabin Joshi; Shady Elsayed; Shafqat Bukhari; Shailendra Magdum; Shungu Ushewokunze; Siddharth Sinha; Simon Lammy; Stana Bojanic; Stewart Griffiths; Teresa Scott; Thomas Carroll; Vasileios Raptopoulos; Vivek Josan; Yasir Chowdhury; Zubair Tahir.
Contributors RJP: study conception, study design, writing of the manuscript and editing of the manuscript. FTA, WCS, AGK, RL, WBL, JJ: study conception, study design and editing of the manuscript. EWD, LW: study design and editing of the manuscript.

Funding This work was supported by Ann Conroy Trust (www.annconroytrust.org). Competing interests None declared.

\section{Patient consent for publication Not required.}

Provenance and peer review Not commissioned; externally peer reviewed.

Supplemental material This content has been supplied by the author(s). It has not been vetted by BMJ Publishing Group Limited (BMJ) and may not have been peer-reviewed. Any opinions or recommendations discussed are solely those of the author(s) and are not endorsed by BMJ. BMJ disclaims all liability and responsibility arising from any reliance placed on the content. Where the content includes any translated material, BMJ does not warrant the accuracy and reliability of the translations (including but not limited to local regulations, clinical guidelines, terminology, drug names and drug dosages), and is not responsible for any error and/or omissions arising from translation and adaptation or otherwise.

Open access This is an open access article distributed in accordance with the Creative Commons Attribution Non Commercial (CC BY-NC 4.0) license, which permits others to distribute, remix, adapt, build upon this work non-commercially, and license their derivative works on different terms, provided the original work is properly cited, appropriate credit is given, any changes made indicated, and the use is non-commercial. See: http://creativecommons.org/licenses/by-nc/4.0/.

\section{ORCID iDs}

Rory J Piper http://orcid.org/0000-0002-6422-5853

Edward W Dyson http://orcid.org/0000-0002-2539-5374

Laurence Watkins http://orcid.org/0000-0003-0678-3774

\section{REFERENCES}

1 Cesmebasi A, Loukas M, Hogan E, et al. The Chiari malformations: a review with emphasis on anatomical traits. Clin Anat 2015;28:184-94.

2 Meadows J, Kraut M, Guarnieri M, et al. Asymptomatic Chiari type I malformations identified on magnetic resonance imaging. J Neurosurg 2000;92:920-6.

3 Chatrath A, Marino A, Taylor D, et al. Chiari I malformation in children-the natural history. Childs Nerv Syst 2019;35:1793-9.

4 Piper RJ, Pike M, Harrington R, et al. Chiari malformations: principles of diagnosis and management. BMJ 2019;365:I1159.

5 Piper RJ, Magdum SA. Chiari 1 malformation and raised intracranial pressure. Childs Nerv Syst 2019;35:1719-25.

6 Chiari H. Ueber Veränderungen des Kleinhirns infolge von Hydrocephalie des Grosshirns. Dtsch med Wochenschr 1891;17:1172-5.

7 Mediratta S, Haden N. Surgeons singing from the same hymn sheet: a proposal for the pre-operative investigation of patients with Chiari malformation. In: Syringomyelia-Chiari 2018 International Symposium, 2018. Available: http://syringomyelia-chiari2018.org/f2.html

8 Schijman E, Steinbok P. International survey on the management of Chiari I malformation and syringomyelia. Childs Nerv Syst 2004;20:341-8.

9 Shweikeh F, Sunjaya D, Nuno M, et al. National trends, complications, and hospital charges in pediatric patients with Chiari malformation type I treated with posterior fossa decompression with and without duraplasty. Pediatr Neurosurg 2015;50:31-7.

10 Gallo P, Sokol D, Kaliaperumal C, et al. Comparison of three different Cranio-Cervical decompression procedures in children with Chiari malformation type I: does the surgical technique matter? Pediatr Neurosurg 2017;52:289-97.

11 Kurzbuch AR, Jayamohan J, Magdum S. Decompressive surgery for Chiari I malformation in children without dural repair: a still effective and safe procedure? Childs Nerv Syst 2019;35:1839-46.

12 McGirt MJ, Attenello FJ, Datoo G, et al. Intraoperative ultrasonography as a guide to patient selection for duraplasty after suboccipital decompression in children with Chiari malformation type I. J Neurosurg Pediatr 2008;2:52-7.

13 Dyson EW, Chari A, Stevens AR, et al. Intracranial pressure guided management of patients with Chiari malformations presenting with headache: a paradigm shift? Fluids Barriers CNS 2015;12:029.

14 Ciaramitaro P, Garbossa D, Peretta P, et al. Syringomyelia and Chiari syndrome Registry: advances in epidemiology, clinical phenotypes and natural history based on a North Western Italy cohort. Ann Ist Super Sanita 2020;56:48-58. 
15 Vedantam A, Mayer RR, Staggers KA, et al. Thirty-day outcomes for posterior fossa decompression in children with Chiari type 1 malformation from the US NSQIP-Pediatric database. Childs Nerv Syst 2016;32:2165-71.

16 Pomeraniec IJ, Ksendzovsky A, Awad AJ, et al. Natural and surgical history of Chiari malformation type I in the pediatric population. J Neurosurg Pediatr 2016;17:343-52.

17 Chari A, Jamjoom AA, Edlmann E, et al. The British Neurosurgical Trainee Research Collaborative: Five years on. Acta Neurochir 2018;160:23-8.

18 Woodfield J, Hoeritzauer I, Jamjoom AAB, et al. Understanding cauda equina syndrome: protocol for a UK multicentre prospective observational cohort study. BMJ Open 2018;8:e025230.
19 Harris PA, Taylor R, Thielke R, et al. Research electronic data capture (REDCap)-a metadata-driven methodology and workflow process for providing translational research informatics support. J Biomed Inform 2009;42:377-81.

20 Nepogodiev D, Chapman SJ, Glasbey J, et al. Determining surgical complications in the overweight (discover): a multicentre observational cohort study to evaluate the role of obesity as a risk factor for postoperative complications in general surgery. BMJ Open 2015;5:e008811.

21 National Research Collaborative \& Association of Surgeons in Training Collaborative Consensus Group. Recognising contributions to work in research Collaboratives: guidelines for standardising reporting of authorship in Collaborative research. Int J Surg 2018;52:355-60. 